\title{
Knowledge of vitamin D and its supplementation among students of northern Poland
}

\section{Przemysław M Waszak ${ }^{1,2}$, Aleksandra Mędza², Janusz Springer ${ }^{3}$, Martyna Zgłobicka², Paula Ogrodnik², Paulina Kalinowska², Piotr Kmieć ${ }^{4}$, Maria Lizakowska-Kmieć ${ }^{5}$, Krzysztof Sworczak ${ }^{4}$, Michał Żmijewski ${ }^{6}$}

\author{
${ }^{1}$ Department of Hygiene \& Epidemiology, Medical University of Gdańsk, Poland \\ ${ }^{2}$ Students' Scientific Association, Histology Department, Medical University of Gdańsk, Poland \\ ${ }^{3}$ Department of Preventive Medicine and Education, Medical University of Gdańsk, Poland \\ ${ }^{4}$ Department of Endocrinology and Internal Medicine, Medical University of Gdańsk, Poland \\ ${ }^{5}$ Endomed Diagnostic Medicine Centre, Gdańsk, Poland \\ ${ }^{6}$ Histology Department, Medical University of Gdańsk, Poland
}

\begin{abstract}
Introduction: Vitamin D deficiency is a worldwide public health problem. The objective of this survey was to assess the undergraduate students' vitamin $D$ status and knowledge about this vitamin. Materials and methods: An online multi-choice survey was designed and launched in Northern Poland (Gdańsk region). The first part of the survey assessed diet, supplementation, UV radiation exposure (UVE) and general health of respondents. The second part was a vitamin D knowledge test (vitamin D optimal level, deficiency-related diseases). 1766 student volunteers responded to the survey: 369 male and 1397 female. Data was divided according to the respondents' sex and university affiliation. Appropriate parametric or non-parametric statistical tests were used with statistical significance set at $p<0.05$. Results: Regular consumption of vitamin D-rich food was high, except for fish (only 18; $22 \%)$. High number of participants did not declare any type of supplementation $(43 ; 44 \%)$ and only occasionally were exposed to UV (77\%; $80 \%)$. The most frequently recognized disease linked to vitamin D was osteoporosis. Medical University students obtained higher test scores $(4,55)$, however this did not correlate with healthy vitamin D habits. Conclusion: Undergraduate medical and non-medical students have unsatisfactory vitamin $D$ status and poor understanding of its function and impact on health, which implies the need for changes in the educational program.

Keywords: vitamin $\mathrm{D} \bullet$ vitamin $\mathrm{D}$ deficiency $\bullet$ knowledge $\bullet$ students
\end{abstract}

\section{Citation}

Waszak PM, Mędza A, Springer J, Zgłobicka M, Ogrodnik P, Kalinowska P, et al. Knowledge of Vitamin D and its Supplementation Among Students of Northern Poland. Eur J Transl Clin Med 2018;1(1):48-56.

DOI: $10.31373 /$ ejtcm/92067 


\section{Introduction}

It is believed that over a billion people worldwide are vitamin $\mathrm{D}$ deficient [1]. The distance from the equator, short summers and cold climate are amongst the major factors for vitamin D deficiency, which puts many European populations at a disadvantage [2]. There is high worldwide prevalence of vitamin D deficiency [3-4]. One population study demonstrated that $84.4 \%$ of participants in Northern Poland (the largest agglomeration is Gdańsk located at $54^{\circ} \mathrm{N}$ ) were vitamin D-deficient during winter $[3,5]$ and this deficiency was only partially compensated by summer exposure to sun light [6].

Vitamin $D$ (cholecalciferol) in its active form $1,25(\mathrm{OH})_{2} \mathrm{D}_{3}$ (calcitriol) is a crucial hormone in the calcium-phosphorus homeostasis. Furthermore, vitamin $D$ regulates more than 1000 genes, thus its biological activity extends far beyond the skeletal system [7-10]. Therefore, vitamin D deficiency has been linked to many illnesses including infections, diabetes, cardiovascular, skin and autoimmune diseases [8, 11-14]. Additionally, several multicenter studies underline the anti-proliferative activity of vitamin $D$ and its analogues against colorectal, breast, prostate and melanoma cancers [15-17].

Vitamin D synthesis begins in skin exposed to UV radiation as a result of photolysis of 7-dehydrocholesterol (pre-vitamin $D_{3}$ ) [8]. It was estimated that an exposure of arms and legs to $0,25-0,50$ minimal erythemal dose (MED) results in formation of 2,000-4,000 IU of vitamin D3 [18]. Dietary supplementation of vitamin D-rich products is limited mainly to oily fish (up to $1,200 \mathrm{lU} / 100 \mathrm{~g}$ ), egg yolk ( 20-50 IU/yolk) and dairy products e.g. milk ( 0.4-1.2 IU/100 $\mathrm{mL}$ ) or cheese ( 7-28 IU/100 g) [1819].

Central European guidelines recommend vitamin $D$ intake between September and April for adults ( $>18$ years) between 800 to $2000 \mathrm{IU} /$ day (20.0-50.0 $\mu \mathrm{g} /$ day). Furthermore, if the summertime sun exposure is insufficient or contraindicated, supplementation should be provided year-round [18].

It is assumed that students, particularly of medicine, have a good understanding of vitamin $D$ and its health benefits. However recent studies reveal that this is not true [20]. According to our literature search, there has not been any study investigating vitamin D knowledge among Polish students.

\section{Materials and methods}

The purpose of this cross-sectional population study was to analyze the vitamin D habits of undergraduates in Northern Poland (Gdańsk area). Specifically, the aim was to assess their diet, vitamin D supplementation, tanning habits, physical activity, vitamin D knowledge and to evaluate their self-assessed state of health. All participants were informed about the purpose of the study on the front page of the survey. Participation was voluntary and the student could withdraw his/her participation at any time. There were no exclusion criteria.

\section{The survey}

An on-line survey was created using Google Forms (Google Inc., California, USA) and conducted out from November 2013 to March 2014. Most of the universities involved in the study had its own student-coordinator for purpose of this study (see: Acknowledgments). University e-mail lists and social media were used to recruit the respondents. The survey contained 17 open or multiple choice questions and was prepared in two identical versions (Polish and English). The survey took approximately 5 minutes to complete.

\section{Vitamin D survey}

\section{Part 1: General information}

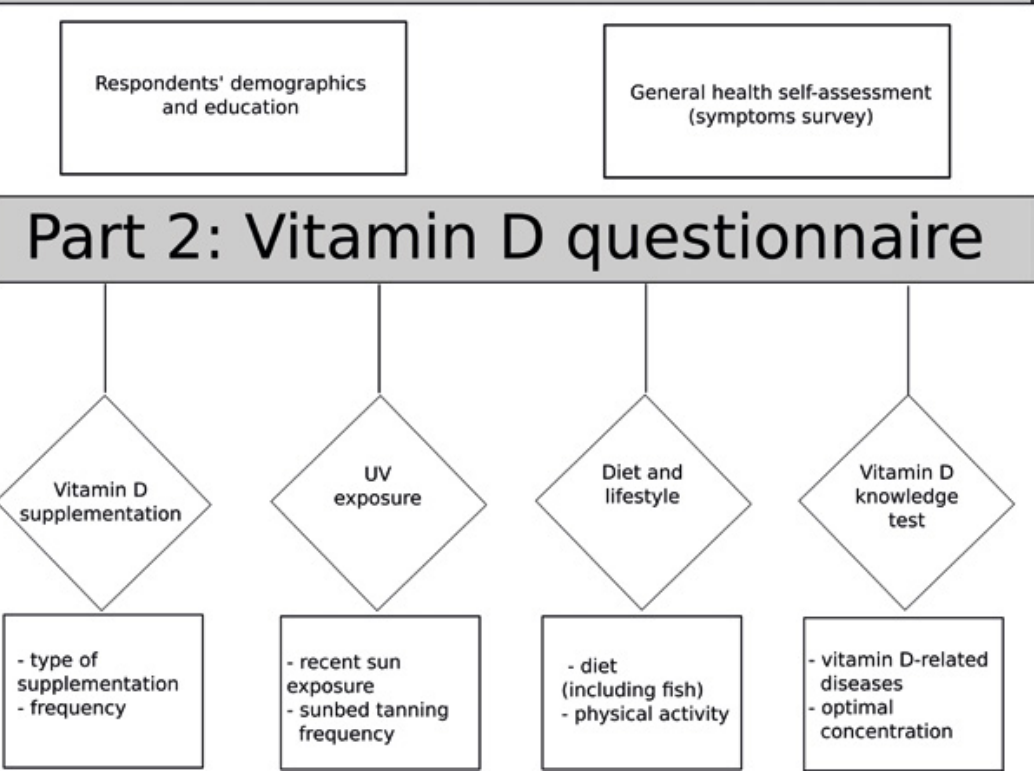

Figure 1. Design of the online Vitamin D survey form .

After providing demographic data, the respondents were asked about the type and frequency of their vitamin D supplementation. Then, the data concerning frequency and the date of last of suntanning outside or tanning salon visit was collected. The dietary preferences were also examined with questions about the 
type and frequency of vitamin D-rich food consumption. The respondents were asked whether they experience common symptoms of vitamin D deficiency. Participants were also asked about their serum $25-\mathrm{OH} \mathrm{D}_{3}$ level, if known.

The final part of the survey assessed the knowledge about diseases related to vitamin $D$ deficiency and the optimal serum level of vitamin D (25-OH-vitamin D). The test part consisted of 2 questions. In question about optimal serum level of $25-\mathrm{OH}$-vitamin $\mathrm{D}$, participant could score 1 point by selecting the proper answer (30-50ng/ml). Second multiple-choice question was a list of 22 diseases, prepared according to current knowledge, including the Central European recommendations and the previously conducted survey in the Gdańsk area. For selecting vitamin $\mathrm{D}$ deficiency-associated diseases participants earned 1 point each (maximum of 20 points). To prevent earning a $100 \%$ score simply by ticking all the available answers, three of the diseases listed in the test were not vitamin D-related (pleuritis, aortic dissection, Down's syndrome.). There was no success/failure threshold value. Vitamin D-related diseases included in test are presented in Figure 2 and were selected according to literature $[4,11,18,21]$.

Prior to launching the survey on-line, 10 participants pilot-tested both the English and Polish versions, to determine whether questions were clearly phrased and to eliminate potential misunderstandings.

\section{Statistical methods}

The database was prepared using MS Excel (Microsoft Office Professional Plus 2010, Redmond, USA). Statistical analyses were performed using the STATISTICA software (StatSoft, Inc. Ver. 10.0, Tulsa, USA). Basic descriptive values (mean, median, standard deviation and range) were calculated. Appropriate tests were performed in order to evaluate the statistical significance of the results (Chi-squared test for qualitative data, parametric or nonparametric tests for quantitative data). Parametric tests were used if the values met the criteria of normal distribution. Nonparametric tests were used if the value had another type of distribution. The U-Mann-Whitney test was used to compare groups not fitting a normal distribution, whereas the t-Student test was performed for the groups with normal distribution. For multiple group comparison, Kruskal-Wallis or ANOVA and tests were used, respectively. The statistical significance was set at $p<0.05$.

\section{Results}

Overall, 1766 completed surveys were received from 369 males and 1397 females (Table 1). Vast majority of the respondents (99\%) were Caucasian. Respondents were Polish (95\%) while 3\% were citizens of Sweden and $2 \%$ came from other countries.

In our survey, regular vitamin D-rich food consumption was defined as consuming each product more than once a week. Overall regular consumption of milk, cheese or eggs was declared by about $60-70 \%$ of re-

Table 1. Demographics of the survey respondents $(\mathrm{N}=1766)$

\begin{tabular}{|c|c|c|}
\hline & FEMALE & MALE \\
\hline SEX (\% [n]) & $\begin{array}{l}79,1 \% \\
{[1397]}\end{array}$ & $\begin{array}{c}20,9 \% \\
{[369]}\end{array}$ \\
\hline $\mathrm{AGE}(\mathrm{am} \pm \mathrm{SD})$ & $22,45( \pm 4,34)$ & $22,77( \pm 3,13)$ \\
\hline $\mathrm{BMI}(\mathrm{am} \pm \mathrm{SD})$ & $21,67( \pm 3,36)$ & $23,92( \pm 3,54)$ \\
\hline \multicolumn{3}{|l|}{$\begin{array}{l}\text { SKIN TYPE } \\
\text { ( } \mathrm{n}[\% \text { of total count }] \text { ) }\end{array}$} \\
\hline $\begin{array}{l}\text { - Fair skin, blue eyes; Burns } \\
\text { easily, tans poorly }\end{array}$ & $\begin{array}{c}409 \\
(23,2 \%)\end{array}$ & $\begin{array}{c}86 \\
(4,9 \%)\end{array}$ \\
\hline $\begin{array}{l}\text { - Light brown skin; Burns } \\
\text { minimally, tans easily }\end{array}$ & $\begin{array}{c}359 \\
(20,4 \%)\end{array}$ & $\begin{array}{c}90 \\
(5,1 \%)\end{array}$ \\
\hline $\begin{array}{l}\text { - Darker white skin; Tans after } \\
\text { initial burn }\end{array}$ & $\begin{array}{c}412 \\
(23,4 \%)\end{array}$ & $\begin{array}{c}150 \\
(8,5 \%)\end{array}$ \\
\hline $\begin{array}{l}\text { Brown skin; Rarely burns, } \\
\text { tans darkly easily }\end{array}$ & $\begin{array}{c}107 \\
(6,1 \%)\end{array}$ & $\begin{array}{c}26 \\
(1,5 \%)\end{array}$ \\
\hline $\begin{array}{l}\text { - Pale white skin, blue/hazel } \\
\text { eyes, blond/red hair; Always } \\
\text { burns, does not tan }\end{array}$ & $\begin{array}{c}104 \\
(5,9 \%)\end{array}$ & $\begin{array}{c}15 \\
(0,8 \%)\end{array}$ \\
\hline $\begin{array}{l}\text { Dark brown or black skin; } \\
\text { Never burns, always tans } \\
\text { darkly }\end{array}$ & $\begin{array}{c}3 \\
(0,2 \%)\end{array}$ & $(0,1 \%)$ \\
\hline
\end{tabular}

$\%$ - percent of respondents; $\mathrm{n}$ - number of respondents; am - arithmetic mean; SD - standard deviation 
Table 2. Respondents' diet, supplementation, UVB exposure and physical activity.

\begin{tabular}{|c|c|c|}
\hline & FEMALE & MALE \\
\hline $\begin{array}{l}\text { SEX } \\
{[\% \text { of total count, }(n)]}\end{array}$ & $\begin{array}{l}79,1 \% \\
(1397)\end{array}$ & $\begin{array}{c}20,9 \% \\
(369)\end{array}$ \\
\hline \multicolumn{3}{|c|}{ VITAMIN D-RICH PRODUCTS REGULAR CONSUMPTION } \\
\hline Milk & $\begin{array}{l}70,7 \% \\
(988) \\
\end{array}$ & $\begin{array}{c}69,1 \% \\
(255)\end{array}$ \\
\hline Fish & $\begin{array}{c}18,0 \% \\
(252)\end{array}$ & $\begin{array}{c}22,2 \% \\
(82)\end{array}$ \\
\hline Cheese & $\begin{array}{l}75,7 \% \\
(1057)\end{array}$ & $\begin{array}{c}76,4 \% \\
(282)\end{array}$ \\
\hline Eggs & $\begin{array}{l}60,7 \% \\
(849)\end{array}$ & $\begin{array}{l}63,7 \% \\
(235)\end{array}$ \\
\hline None of them in regular diet & $\begin{array}{c}3,3 \% \\
(46)\end{array}$ & $\begin{array}{c}3,8 \% \\
(14)\end{array}$ \\
\hline \multicolumn{3}{|c|}{ SUPPLEMENTS USAGE } \\
\hline Vitamin D pills & $\begin{array}{c}5,4 \% \\
(75)\end{array}$ & $\begin{array}{c}4,6 \% \\
(17)\end{array}$ \\
\hline Multivitamin & $\begin{array}{c}23,3 \% * \\
(326)\end{array}$ & $\begin{array}{c}29,5 \% * \\
(109)\end{array}$ \\
\hline Cod-liver oil & $\begin{array}{c}5,8 \% * \\
(82)\end{array}$ & $\begin{array}{c}9,7 \% * \\
(36)\end{array}$ \\
\hline Vitamin D + calcium pills & $\begin{array}{c}1,5 \% * \\
(22)\end{array}$ & $\begin{array}{c}3,7 \% * \\
(14)\end{array}$ \\
\hline Calcium pills & $\begin{array}{c}4,1 \% \\
(58)\end{array}$ & $\begin{array}{c}5,1 \% \\
(19)\end{array}$ \\
\hline \multicolumn{3}{|c|}{ SUPPLEMENTATION FREQUENCY } \\
\hline - Daily & $\begin{array}{c}65,4 \% * \\
(493)\end{array}$ & $\begin{array}{c}56,6 \% * \\
(115)\end{array}$ \\
\hline - Weekly & $\begin{array}{c}15,2 \% \\
(115)\end{array}$ & $\begin{array}{c}14,3 \% \\
(29)\end{array}$ \\
\hline - Monthly or rarely & $\begin{array}{c}19,4 \% * \\
(146)\end{array}$ & $\begin{array}{c}29,1 \% * \\
(59)\end{array}$ \\
\hline No supplementation & $\begin{array}{c}44,0 \% \\
(615) \\
\end{array}$ & $\begin{array}{c}43,3 \% \\
(160)\end{array}$ \\
\hline \multicolumn{3}{|c|}{ TANNING HABITS } \\
\hline $\begin{array}{l}\text { Last sunbathing episode after } \\
\text { October }\end{array}$ & $\begin{array}{c}10,6 \% * \\
(142)\end{array}$ & $\begin{array}{c}6,3 \% * \\
(21)\end{array}$ \\
\hline \multicolumn{3}{|l|}{ Tanning frequency } \\
\hline - Weekly & $\begin{array}{c}3,5 \% * \\
(49)\end{array}$ & $\begin{array}{c}6,5 \% * \\
(24)\end{array}$ \\
\hline - Monthly & $\begin{array}{c}20,8 \% * \\
(289) \\
\end{array}$ & $\begin{array}{c}13,5 \% * \\
(50)\end{array}$ \\
\hline - Rarely & $\begin{array}{l}75,6 \% \\
(1050)\end{array}$ & $\begin{array}{l}79,9 \% \\
(295)\end{array}$ \\
\hline \multicolumn{3}{|c|}{ PHYSICAL ACTIVITY - FREQUENCY } \\
\hline - Weekly & $\begin{array}{c}34,6 \% * \\
(482)\end{array}$ & $\begin{array}{c}45,1 \% * \\
(165)\end{array}$ \\
\hline - Monthly & $\begin{array}{c}42,4 \% * \\
(591)\end{array}$ & $\begin{array}{c}35,2 \% * \\
(129)\end{array}$ \\
\hline - Rarely & $\begin{array}{c}22,9 \% * \\
(319)\end{array}$ & $\begin{array}{c}19,6 \% * \\
(72)\end{array}$ \\
\hline \multicolumn{3}{|c|}{ VITAMIN D DIAGNOSTICS } \\
\hline $\begin{array}{l}\text { Ever measured their } 25(\mathrm{OH}) \text { vitamin } \\
\text { D level }\end{array}$ & $\begin{array}{c}5,0 \% * \\
(70)\end{array}$ & $\begin{array}{l}2,5 \% * \\
(9)\end{array}$ \\
\hline
\end{tabular}

${ }^{*} p<0,05$

spondents of both sexes (Table 2). However, regular fish consumption was reported only by $18 \%$ of women and $22 \%$ of men.
Up to $44 \%$ of participants declared at least one type of vitamin $D$ supplementation, with multivitamins being most common. More males than females selected gen- 
eral intake of each form of supplement (except vitamin D pills). However, in this same group only $56 \%$ admitted taking their supplements daily (Table 2).

Analysis showed a significantly higher usage of sunbed tanning during the autumn/winter season among women, but this exposure was attributed to only $10 \%$ of studied female respondents. Physical activity was more frequent in the male group.

Only $5 \%$ of the studied women and $2.5 \%$ of the men measured their $25-\mathrm{OH}$-vitamin $\mathrm{D}$ level in the past (Table 2). Furthermore, the difference was even more significant when Polish and foreign students were compared ( $4 \%$ vs $10 \%$, respectively; $p<0,05$ ). Among those who have tested their $25-\mathrm{OH}$-vitamin $\mathrm{D}$ serum level,

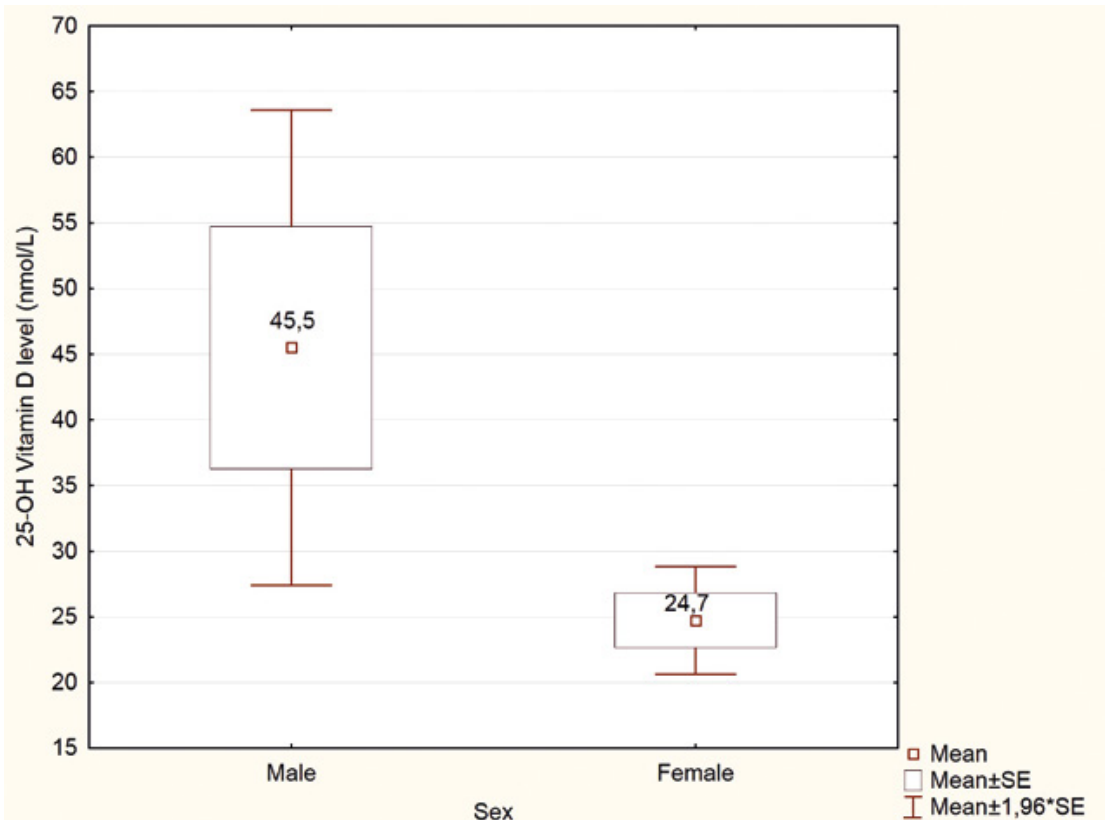

Figure 2. Respondents' self-reported serum 25(OH)D concentration, (nmol/L). p<0,05 males tend to report significantly higher laboratory values than females (45.6 vs $24.7 \mathrm{nmol} / \mathrm{L} ; \mathrm{p}<0.05 ; \mathrm{n}=79$ ) (Figure 2).

Respondents who declared supplementation reported a higher rate of muscle weakness and problems with concentration, whereas those who have been exposed to UV during autumn or winter have significantly less problems with concentration (Table 3).

Medical University students earned significantly higher scores on the vitamin D-related knowledge test compared to those from other universities (mean result 4.55 points out of maximum 20 points). On subsequent positions placed students from the University of Social Sciences and Humanities (3.59) and the University of Business and Administration in Gdynia (3.22). The lowest mean scores were found among the students of the Gdańsk University of Technology or the Academy of Music in Gdańsk (both $\sim 2.6$ points). Despite the highest test performance, students of Medical University of Gdańsk were the group with the biggest proportion of non-supplementing students (48.7\%). Lack of supplementation was also highly prevalent among the Naval Academy students $-47.5 \%$ (see Table 4).

The most commonly selected as vitamin D-related diseases were as follows: osteoporosis (48\%), depression (43\%) and rheumatoid arthritis (30\%). A minority of participants recognized autoimmune or pulmonary diseases as vitamin D-related diseases (Figure 3).

Table 3. Vitamin D habits and nonspecific deficiency symptoms.

\begin{tabular}{|c|c|c|c|c|c|c|}
\hline $\begin{array}{l}\text { Subjective } \\
\text { symptoms }\end{array}$ & $\begin{array}{l}\text { Supplemen- } \\
\text { ting stu- } \\
\text { dents }\end{array}$ & $\begin{array}{l}\text { Non-sup- } \\
\text { plemen- } \\
\text { ting stu- } \\
\text { dents }\end{array}$ & $\begin{array}{l}\text { Autumn } \\
\text { and winter } \\
\text { tanning } \\
\text { students }\end{array}$ & $\begin{array}{l}\text { Non-autumn } \\
\text { and winter } \\
\text { tanning } \\
\text { students }\end{array}$ & $\begin{array}{l}\text { Students with } \\
\text { vitamin D -rich } \\
\text { product in } \\
\text { regular diet }\end{array}$ & $\begin{array}{c}\text { Students } \\
\text { without } \\
\text { vitamin D -rich } \\
\text { product in } \\
\text { regular diet }\end{array}$ \\
\hline $\begin{array}{l}\text { Muscular pain } \\
{[\% \text { of total }} \\
\text { count, }(n)]\end{array}$ & $\begin{array}{c}47,5 \% \\
(466)\end{array}$ & $\begin{array}{l}44,2 \% \\
(339)\end{array}$ & $\begin{array}{c}48,1 \% \\
(78)\end{array}$ & $\begin{array}{l}45,9 \% \\
(686)\end{array}$ & $\begin{array}{l}46,2 \% \\
(779)\end{array}$ & $\begin{array}{c}43,3 \% \\
(26)\end{array}$ \\
\hline $\begin{array}{l}\text { Muscle } \\
\text { weakness }\end{array}$ & $\begin{array}{c}37,9 \% * \\
(373)\end{array}$ & $\begin{array}{c}33,0 \% * \\
(254)\end{array}$ & $\begin{array}{c}32,7 \% \\
(53)\end{array}$ & $\begin{array}{c}36,4 \% \\
(546)\end{array}$ & $\begin{array}{c}35,6 \% \\
(604)\end{array}$ & $\begin{array}{c}38,3 \% \\
(23)\end{array}$ \\
\hline $\begin{array}{l}\text { Problems with } \\
\text { concentration }\end{array}$ & $\begin{array}{c}57,2 \% * \\
(560) \\
\end{array}$ & $\begin{array}{c}52,5 \% * \\
(402)\end{array}$ & $\begin{array}{c}46,9 \% * * \\
(76)\end{array}$ & $\begin{array}{c}55,7 \% * * \\
(830)\end{array}$ & $\begin{array}{c}55,1 \% \\
(927)\end{array}$ & $\begin{array}{c}58,3 \% \\
(35)\end{array}$ \\
\hline Bad mood & $\begin{array}{c}62,7 \% \\
(617) \\
\end{array}$ & $\begin{array}{c}59,7 \% \\
(460) \\
\end{array}$ & $\begin{array}{c}58,0 \% \\
(94) \\
\end{array}$ & $\begin{array}{c}61,8 \% \\
(927) \\
\end{array}$ & $\begin{array}{l}61,7 \% \\
(1046) \\
\end{array}$ & $\begin{array}{c}51,7 \% \\
(31)\end{array}$ \\
\hline $\begin{array}{l}\text { Frequent } \\
\text { infections in } \\
\text { the last year }\end{array}$ & $\begin{array}{c}19,2 \% \\
(190)\end{array}$ & $\begin{array}{l}16,3 \% \\
(126)\end{array}$ & $\begin{array}{c}17,8 \% \\
(29)\end{array}$ & $\begin{array}{l}17,7 \% \\
(267)\end{array}$ & $\begin{array}{c}17,8 \% \\
(303)\end{array}$ & $\begin{array}{c}21,7 \% \\
(13)\end{array}$ \\
\hline
\end{tabular}

** and ${ }^{*}$ statistically significant differences between two groups $(p<0,05)$ 
Table 4. Results by the respondents' university affiliation.

\begin{tabular}{|c|c|c|c|c|c|c|}
\hline University & $\begin{array}{c}\text { Respon- } \\
\text { dents per- } \\
\text { cent }[\%] \\
\text { Number }[\mathrm{n}]\end{array}$ & $\begin{array}{l}\text { Official } \\
\text { number of } \\
\text { students } \\
\text { (year) }\end{array}$ & $\begin{array}{l}\text { Sample size } \\
\text { as percent of } \\
\text { total student } \\
\text { enrollment }\end{array}$ & $\begin{array}{c}\text { Mean } \\
\text { Vitamin D } \\
\text { Test } \\
\text { Score } \\
\end{array}$ & $\begin{array}{l}\text { Lack of vita- } \\
\text { min D suple- } \\
\text { mentation }\end{array}$ & $\begin{array}{c}\text { Frequent } \\
\text { sun/sunbed } \\
\text { tanning }\end{array}$ \\
\hline $\begin{array}{l}\text { Medical University of } \\
\text { Gdansk }\end{array}$ & $\begin{array}{c}45,4 \% \\
(801) \\
\end{array}$ & $\begin{array}{c}6505 \\
(2011) \\
\end{array}$ & $12,3 \%$ & $4,55^{*}$ & $48,9 \%$ & $26,5 \%$ \\
\hline University of Gdansk & $\begin{array}{c}24,5 \% \\
(433) \\
\end{array}$ & $\begin{array}{l}28625 \\
(2010)\end{array}$ & $1,5 \%$ & 2,96 & $41,1 \%$ & $19,6 \%$ \\
\hline $\begin{array}{l}\text { Gdansk University of } \\
\text { Technology }\end{array}$ & $\begin{array}{c}10,9 \% \\
(193)\end{array}$ & $\begin{array}{l}25000 \\
(2010)\end{array}$ & $0,7 \%$ & $2,66 * *$ & $42,2 \%$ & $17,7 \%$ \\
\hline $\begin{array}{l}\text { University of Business } \\
\text { and Administration in } \\
\text { Gdynia }\end{array}$ & $\begin{array}{c}4,6 \% \\
(82)\end{array}$ & $\begin{array}{l}3000 \\
(2014)\end{array}$ & $2,7 \%$ & 3,32 & $36,6 \%$ & $34,1 \%$ \\
\hline $\begin{array}{l}\text { Gdynia Maritime } \\
\text { University }\end{array}$ & $\begin{array}{c}3,5 \% \\
(62)\end{array}$ & $\begin{array}{c}6633 \\
(2011)\end{array}$ & $0,9 \%$ & 3,16 & $37,1 \%$ & $27,8 \%$ \\
\hline Polish Naval Academy & $\begin{array}{c}2,3 \% \\
(40)\end{array}$ & $\begin{array}{c}4026 \\
(2014)\end{array}$ & $1,0 \%$ & 2,77 & $47,5 \%$ & $42,5 \%$ \\
\hline $\begin{array}{l}\text { University of Social } \\
\text { Sciences } \\
\text { and Humanities }\end{array}$ & $\begin{array}{c}2,1 \% \\
(37)\end{array}$ & ND & - & $3,59 * *$ & $32,4 \%$ & $24,3 \%$ \\
\hline $\begin{array}{l}\text { Gdansk University of } \\
\text { Physical Education } \\
\text { and Sport }\end{array}$ & $\begin{array}{l}1,9 \% \\
(34)\end{array}$ & $\begin{array}{l}3060 \\
(2011)\end{array}$ & $1,1 \%$ & 2,82 & $29,4 \%$ & $41,9 \%$ \\
\hline $\begin{array}{l}\text { Academy of Fine Arts } \\
\text { in Gdansk }\end{array}$ & $\begin{array}{c}1,1 \% \\
(19)\end{array}$ & $\begin{array}{c}820 \\
(2011)\end{array}$ & $2,3 \%$ & 2,82 & $44,4 \%$ & $16,6 \%$ \\
\hline $\begin{array}{l}\text { Academy of Music in } \\
\text { Gdansk }\end{array}$ & $\begin{array}{c}0,9 \% \\
(16)\end{array}$ & $\begin{array}{c}782 \\
(2011) \\
\end{array}$ & $2,0 \%$ & 2,62 & $37,5 \%$ & $6,2 \%$ \\
\hline ALL RESPONDENTS & 1709 & - & - & 3,69 & $43,5 \%$ & $23,5 \%$ \\
\hline
\end{tabular}

${ }^{*} p<0,05$ comparing to all other groups; ${ }^{* *} p<0,05$ for difference between these two groups; ND- no data

Due to the large subset of Medical University of Gdansk students $(n=801)$, this group was analyzed also by the degree program (Figure 4). The highest test scores were observed among the Dietetics students (mean $7.51 ; n=49)$, followed by the Public Health (5.60; $\mathrm{n}=10$ ) students. Students of the MD program, with mean score of $4.75(n=335)$ for the Polish students and $4.24(n=110)$ for the foreign students, scored similarly to the overall average of the Medical University of Gdańsk participants (mean 4.55, $\mathrm{n}=801$ ).

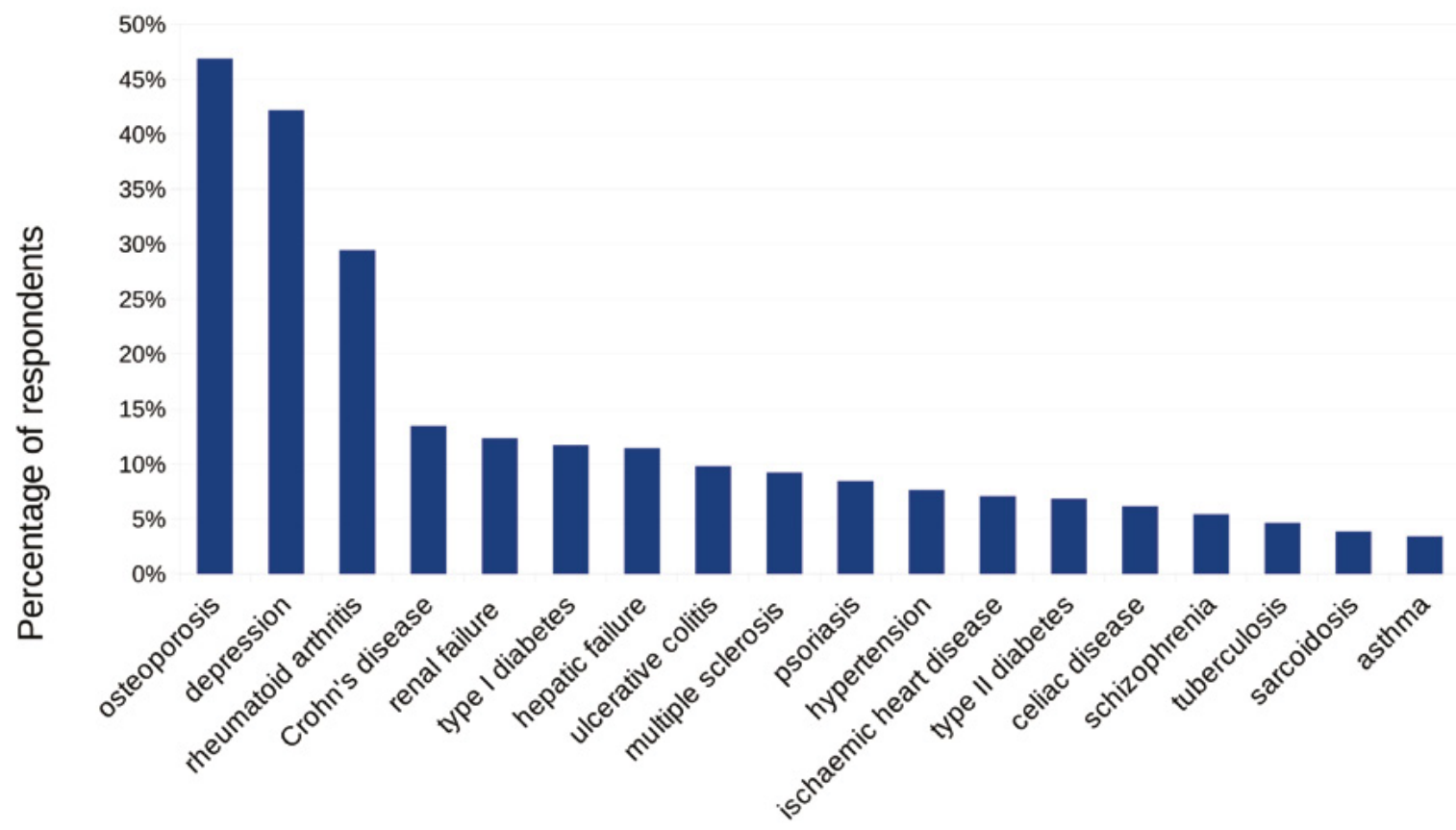

Figure 3. Most frequently selected vitamin D-related diseases in the knowledge test $(N=1710)$ 


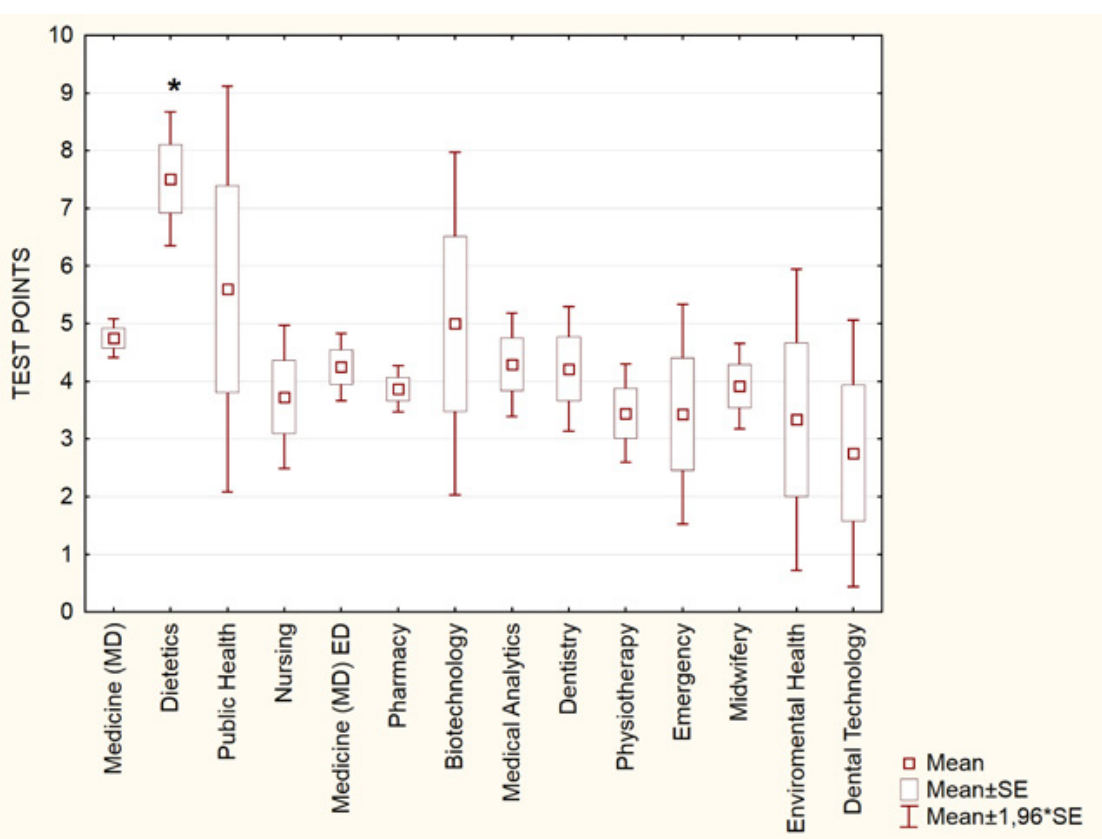

Figure 4. Vitamin D knowledge test performance among Medical University of Gdańsk students, by degree program.

\section{Discussion}

The undergraduate students surveyed in this cross-sectional study represent various medical education backgrounds. Their vitamin $D$ knowledge and lifestyle attitude were assessed. Based on Central European recommendations [18], the main sources of vitamin $D$ are: UV exposure, supplementation and diet. While sun exposure in Europe is limited to certain months, sufficient food and supplement intake should be achieved for most of the year $[1,18]$.

Undergraduate students around the world generally have poor knowledge about nutritional sources of vitamin D. According to a study from Bangladesh, nearly $20 \%$ of pharmacy students failed to mention at least one vitamin D-rich product [22]. A study performed among Polish undergraduate students based on their dietary records (200 participants, southern Poland) showed that both sexes have insufficient daily vitamin D consumption (mean values were $2.45 \mu \mathrm{g}$ for women and $3,97 \mu \mathrm{g}$ for men) [23]. Another Polish study reported even smaller values: $2.2 \mu \mathrm{g}$ (men) and 3.5 $\mu \mathrm{g}$ (women) [24]. Similarly, a US survey revealed that only $22 \%$ of the female and $47 \%$ of the male students attained the adequate intake for vitamin D [25]. Our results are similar, as the percentage of students consuming vitamin D-rich products was low. Additionally, only a minority of our respondents reported regular fish consumption.

A large cross-sectional study of Northern Poland population has shown a high prevalence of vitamin $D$ deficiency during the winter months $(84.4 \%$ of participants had serum 25-OH-vitamin D level below $20 \mathrm{ng} / \mathrm{mL}$ ) [4]. In the same paper, the authors pointed out that daily supplementation of vitamin $D$ is the strongest independent factor that increases the 25-OH-vitamin D serum level with odds ratio 4.57 (2.34-8.94) [4]. In our study, lack of any form of supplementation was observed in nearly half of studied participants.

Kmieć et al. also tested population vitamin $\mathrm{D}$ status with regard to UVE. Mean 25-OH vitamin $D$ concentration among the participants in autumn (after months with high sun exposure) was $22.8 \pm 7.9 \mathrm{ng} / \mathrm{mL}[6]$ and $14.3 \pm 6.6 \mathrm{ng} / \mathrm{mL}$ during winter [4]. Vast majority of our participants tended to avoid UVE in any form (75-80\% of them reported "rarely" UVE), both during autumn or winter months. Only a part of respondents reported regular (weekly or monthly) exposure to sunlight or use of sunbeds.

A Pakistani survey of medical students showed higher usage of vitamin D supplements among those suffering from fatigue and muscular pain [26].Concerning our study participants, it is not clear whether such non-specific symptoms can be relate to actual vitamin $D$ deficiency or to another medical condition. Table 4 shows nearly similar prevalence of these symptoms (except problems with concentration) both in vitamin D supplementing and non-supplementing students.

Research on a large sample of Canadian students' ( $\mathrm{N}=1088$ ), reported poor knowledge test scores: $26 \%$ on vitamin D sources, $23 \%$ on factors affecting its level and $37 \%$ on its health effects [20]. Only $8 \%$ of the students knew the recommended vitamin D intake [20]. A survey from Pennsylvania found no association between students' knowledge of bone health with vitamin $D$ or calcium intake ( $r=0.04$, NS) [25]. Survey from China reveals, that despite students' high test performance (up to $87.3 \%$ positive answers), majority of them (66.8\%) never or seldom increased sun exposure [27]. Similarly in our respondents: some groups had good general knowledge about the consequences of vitamin D deficiency, but they also had high ratio of non-supplementing and non-UV exposed participants. This observation concerned particularly Medical University students.

The role of vitamin D deficiency in development of osteoporosis is well-recognized in general population [28-29]. However it is still not clear how vitamin D influences depression, as multiple studies showed strong 
though not consistent associations [30-32]. Turkish first-year medical students most frequently recognized osteoporosis as a vitamin D-deficiency related disease [33]. Among our respondents, both osteoporosis and depression were the most frequently chosen answers.

The main limitations of our study is data uncertainty. Without the participants' detailed dietary records and measured 25-OH-vitamin D serum levels, it is impossible to objectively assess one's vitamin D status. There can be potential respondent bias as well, because internet-based surveys are prone to interference due to the internet's distracting and anonymous environment [34]. Our study group could have been adjusted to fully represent the general population. As in our study, the student population of Polish universities is very predominantly female [35]. Furthermore, apart from the Medical University of Gdańsk, the proportional sample size of each university's cohort was similar (Table 4).
Our results suggest that despite the growing number of evidence about vitamin D's health benefits, students from the Gdańsk area have low vitamin D status along with poor understanding of vitamin D deficiency. Given the individual and public health consequences of vitamin D deficiency, it should be worthwhile to launch an effective informational campaign and to implement the university curricula with the latest evidence and recommendations on vitamin D. Vitamin D education and supplementation should be also added to clinical practice, with special regard to primary healthcare providers.

Online educational programs could be effective in improving knowledge as well as vitamin D awareness software [36-37]. Investment in the healthy habits of individuals should be one of the principal long-term goals of healthcare policy makers. Developing public health policies focused on vitamin $D$ should be regarded as primary prevention, which eventually would enhance the health status of the entire population.

\section{References}

1. Holick MF, Chen TC. Vitamin D deficiency: a worldwide problem with health consequences. Am J Cinical Nutr. 2008;87(4):1080S-6S.

2. Nowson CA, Diamond TH, Pasco JA, Mason RS, Sambrook PN, Eisman JA. Vitamin D in Australia. Issues and recommendations. Aust Fam Physician. 2004;33(3):133-8.

3. Slominski RM, Zmijewski M a., Slominski AT. The role of melanin pigment in melanoma. Exp Dermatol. 2015;24(4):258-9.

4. Kmieć P, Żmijewski M, Waszak P, Sworczak K, Lizakowska-Kmieć M. Vitamin D deficiency during winter months among an adult, predominantly urban, population in northern Poland. Endokrynol Pol. 2013;65(2):105-13.

5. Kmieć P, Sworczak K. Vitamin D deficiency in early autumn among predominantly non-elderly, urban adults in Northern Poland ( $54{ }^{\circ} \mathrm{N}$ ). Postep Hig Med Dosw. 2015;69:918-24.

6. Kmieć $\mathrm{P}$, Żmijewski M, Lizakowska-Kmieć $\mathrm{M}$, Sworczak K. Widespread vitamin $\mathrm{D}$ deficiency among adults from northern Poland ( $\left.54^{\circ} \mathrm{N}\right)$ after months of low and high natural UVB radiation. Endokrynol Pol. 2015;66(1):30-8.

7. Carlberg C, Seuter S, de Mello VDF, Schwab U, Voutilainen S, Pulkki K, et al. Primary Vitamin D Target Genes Allow a Categorization of Possible Benefits of Vitamin D3 Supplementation. PLoS One. 2013;8(7):e71042.

8. Wierzbicka J, Piotrowska A, Żmijewski MA. The renaissance of vitamin D. Acta Biochim Pol. 2014;61(4): 679-686.

9. Högler W. Complications of vitamin D deficiency from the foetus to the infant: One cause, one prevention, but who's responsibility? Best Pract Res Clin Endocrinol Metab. 2015;29(3):385-98.

10. Konishi F, Harrison SL. Vitamin D for adults. J Nutr Educ. 1979;11(3):120-2.

11. Holick MF. Vitamin D: Importance in the prevention of cancers, type 1 diabetes, heart disease, and osteoporosis. Am J Clin Nutrition. 2004;79(3):362-71.

12. Joseph AJ, George B, Pulimood AB, Seshadri MS, Chacko A. $25(\mathrm{OH})$ vitamin D level in Crohn's disease: Association with sun exposure \& disease activity. Indian J Med Res. 2009;130(2):133-7.

13. Carvalho LSF, Sposito AC. Vitamin D for the prevention of cardiovascular disease: Are we ready for that? Atherosclerosis. 2015;241(2):72940.

14. Piotrowska A, Wierzbicka J, Zmijewski MA. Vitamin D in the skin physiology and pathology. Acta Biochim Pol. 2016;63(1):17-29.

15. Holick MF. Vitamin D and sunlight: strategies for cancer prevention and other health benefits. Clin J Am Soc Nephrol. 2008;3(5):1548-54.

16. Gorham ED, Garland CF, Garland FC, Grant WB, Mohr SB, Lipkin M, et al. Optimal Vitamin D Status for Colorectal Cancer Prevention. A Quantitative Meta Analysis. Am J Prev Med. 2007;32(3):210-6.

17. Szyszka P, Zmijewski M, Slominski A. New vitamin D analogs as potential therapeutics in melanoma. Expert Rev Anticancer Ther. 2012;12(5):585-99.

18. Płudowski P, Karczmarewicz E, Bayer M, Carter G, Chlebna-Sokół D, Czech-Kowalska, Justyna Dębski R, et al. Practical guidelines for the supplementation of vitamin D and the treatment of deficits in Central Europe - recommended vitamin D intakes in the general population and groups at risk of vitamin D deficiency. Endokrynol Pol. 2013;64(4):480-93.

19. Hyppönen E, Boucher BJ, Berry DJ, Power C. 25-hydroxyvitamin D, IGF-1, and metabolic syndrome at 45 years of age A cross-sectional study in the 1958 british birth cohort. Diabetes. 2008;57(2):298-305.

20. Boland S, Irwin JD, Johnson AM. Research Brief A Survey of University Students ' Vitamin D - Related Knowledge. J Nutr Educ Behav. 2015;47(1):99-103. 
21. Rosen CJ, Adams JS, Bikle DD, Black DM, Demay MB, Manson JE, et al. The nonskeletal effects of vitamin D: an Endocrine Society scientific statement. Endocr Rev. 2012;33(3):456-92.

22. Uddin R, Huda NH, Jhanker YM, Jesmeen T, Imam MZ, Akter S. Awareness regarding the importance of calcium and vitamin D among the undergraduate pharmacy students in Bangladesh. BMC Res Notes. 2013;6(1):134.

23. Gil M, Głodek E, Rudy M. Ocena spożycia witamin i składników mineralnych w całodziennych racjach pokarmowych studentów uniwersytetu rzeszowskiego. Rocz Panstw Zakl Hig. 2012;63(4):441-6.

24. Seidler T, Szczuko M. Ocena sposobu żywienia studentów akademii rolniczej w szczecinie w 2006 roku. Cz. I. Spożycie wybranych składników odżywczych i stan odżywienia. Rocz Panstw Zakl Hig PZH. 2009;60(1):59-64.

25. Lacey JM, Stolfo MM, Rieger RH. Fortified soymilk's potential to improve vitamin D intakes of college students. Nutr Res. 2004;24(2):147-55.

26. Qureshi AZ, Zia Z, Gitay MN, Khan MU, Khan MS. Attitude of future healthcare provider towards vitamin D significance in relation to sunlight exposure. Saudi Pharm J. 2015;23(5):523-527.

27. Gao Q, Liu G, Liu Y. Knowledge, attitude and practice regarding solar ultraviolet exposure among medical university students in Northeast China. J Photochem Photobiol B Biol. 2014;140:14-9.

28. McKean H, Looker S, Hartmann LC, Hayman SR, Kaur JS, McWilliams RR, et al. Are Cancer Survivors/Patients Knowledgeable About Osteoporosis? Results from a Survey of 285 Chemotherapy-treated Cancer Patients and Their Companions. J Nutr Educ Behav. 2008;40(3):144-8.

29. Costa-Paiva L, Gomes DC, Morais SS, Pedro AO, Pinto-Neto AM. Knowledge about osteoporosis in postmenopausal women undergoing antiresorptive treatment. Maturitas. 2011;69(1):81-5.

30. Schneider B, Weber B, Frensch A, Stein J, Fritze J. Vitamin D in schizophrenia, major depression and alcoholism. J Neural Transm. 2000;107(7):839-42.

31. Anglin RES, Samaan Z, Walter SD, McDonald SD. Vitamin D deficiency and depression in adults: systematic review and meta-analysis. Br J Psychiatry. 2013;202(2):100-7.

32. Gowda U, Mutowo MP, Smith BJ, Wluka AE, Renzaho AMN. Vitamin D supplementation to reduce depression in adults: Meta-analysis of randomized controlled trials. Nutrition. 2015;31(3):421-9.

33. Fakültesi T, Osteoporoz Ö, Durmufl D, Akyol Y, Ulus Y, Tander B. Awareness and Sources of Information About Osteoporosis Among Medical Students. Turk J Osteoporos. 2009;15(2):43-7.

34. Evans JR, Mathur A. The value of online surveys. Internet Res. 2005;15(2):195-219.

35. Ministerstwo Nauki i Szkolnictwa Wyższego. Szkolnictwo wyższe w polsce 2013. 1st ed. Warszawa: Ministerstwo Nauki i Szkolnictwa Wyższego; 2013.

36. Goodman S, Morrongiello B, Randall Simpson J, Meckling K. Vitamin D Intake Among Young Canadian Adults: Validation of a Mobile Vitamin D Calculator App. J Nutr Educ Behav. 2015;47(3):242-247.e1.

37. Bonevski B, Magin P, Horton G, Bryant J, Randell M, Kimlin MG. An internet based approach to improve general practitioners' knowledge and practices: The development and pilot testing of the "ABC's of vitamin D" program. Int J Med Inform. 2015;84(6):413-22. 